\title{
Commentary on "Near-Death Experiences with Hallucinatory Features"
}

\author{
Peter Fenwick, M.D., F.R.C.Psych. \\ Institute of Psychiatry, King College, London, U.K. \\ Mental Health Group, University of Southampton
}

\begin{abstract}
In this response to Keith Augustine's paper, I discuss the question of the nature and causation of near-death experiences (NDEs) with hallucinatory features. The attribution of hallucinations to either a brain mechanism or a peek into the afterworld raises fundamental questions about both the epistemology and ontology of our neuroscience, and of our scientific models of an afterlife. It also raises questions about the physiological state of the brain giving rise to NDEs that arise in very different situations and are clearly unlikely to have a unitary cause. These fundamental questions can be answered only in proper prospective trials when both the brain physiology and psychological variables of the experiencer are known.
\end{abstract}

$K E Y$ WORDS: near-death experience; out-of-body experience; epistemology; ontology; models of consciousness.

Keith Augustine's paper examines what he calls "near-death experiences with hallucinatory features," and asks whether these features are brain-generated hallucinations or a glimpse into the afterlife. The paper is essentially about the epistemology, or the nature and grounds of our knowledge, and the ontology of near-death experience (NDEs). Without a full understanding and description of the philosophical grounds on which this article implicitly draws, it is

Peter Fenwick, M.D., F.R.C.Psych., is Honorary Senior Lecturer at the Institute of Psychiatry, Kings College, London, and associated with the Mental Health Group at the University of Southampton. Reprint requests should be addressed to Dr. Fenwick at the Institute for Psychiatry, deCrespigny Park Road, London S.E.5, United Kingdom; email: peter_fenwick@compuserve.com. 
not possible to attempt an answer to the questions it puts forward (Beauregard, 2007; Schwartz, Stapp, and Beauregard, 2005).

First, it asks questions about hallucinations. A hallucination is defined as a sensory experience that is not based on a physical perception and that is not shared between people. Thus the auditory hallucinations of schizophrenia that can be seen to correlate with activations in the auditory cortex are private to the individual and not based on any external sensations. If one was looking at a functional magnetic resonance imaging (fMRI) image of a hallucinating schizophrenic brain and was able to see this in real time, one could certainly see a reflection of the experience, but this would not change our definition of it as a hallucination, as we would not have access by direct perception to the experience itself (Halligan, 2002).

The ontology and epistemology of a brain-based hallucination is fairly clear. It is based on the assumption that brains exist in a physical world and that internal stimulation of brains gives rise to a set of phenomena that we call hallucinatory perceptions, and that these can then be divided up as they are by neuroscience. It must be recognized, however, that even hallucinations that we think we understand are ephemeral, in the sense that they are subjective descriptions that are given to us. We have to take on trust that the person reporting a hallucination is experiencing this. A hallucination has no objective reality, but is simply a subjective description of the experience by the experiencer. We have learned to codify what people report into categories and thus the hallucinations have some quasicategorical status, but that is as far as we can go in our understanding of their nature (Beauregard, 2007; Schwartz, Stapp, and Beauregard, 2005; Velmans, 2002a, 2002b).

When we come to glimpses of the afterlife, the situation becomes much more difficult, so a precise definition is required. What is an afterlife? Presumably Augustine makes the assumption that an afterlife is a continuation of consciousness after the physical death of the brain. This raises profound questions as to the nature of the physical world in which we live and how we obtain information about it. Unfortunately, Augustine does not define an afterlife and thus it is difficult to know what structure he is suggesting as the basis for the afterlife experiences. Is he suggesting that the afterlife is composed of matter and that it is a realm into which one can peek, rather like a glimpse through an open door? And is he suggesting that the rules which apply to this peek are similar to the rules which apply when 
looking through an open door in this world? It would seem to me that if this is his assumption, this needs to be stated. Another view of the afterlife could be that it is somehow a continuation of personal consciousness. This would imply that the memories, psychical structures, and understanding of the experiencer continue in some dimensional space where consciousness exists after death. If this were so, then this afterlife would be very similar to this life, and I do not know how one could easily draw a distinction between the two. There are, of course, other alternative models of the nature of the afterlife that have been reviewed elsewhere (Fontana, 2004), but the ones I have taken span most other views.

The question that Augustine sets us is whether the private experience of an individual to which we do not have access, except by description, should be allocated to one of the two classes of experience described above: brain-based hallucinations, or a peek into the afterlife. It seems to me that this quest is doomed from the outset, because there are no clear ways that we can differentiate between the two. There is not, it seems to me, a falsifiable hypothesis. Both sets of experiences are hallucinations by definition: that is, they are subjective, not shared, and have no external sensory basis. They would seem to be brain-based, except in the cardiac arrest model described below. But having said that, how can we go on to decide that they are not also a peek into some other reality, say an afterlife? We have to be guided, as one does for any sensory experience, by the meaning that the experiencer attaches to it. It is easy then to relate them to some common reality that we all share.

For example, if my patient tells me that he or she is being followed by agents of the British Security Service, commonly known as Military Intelligence, Section 5 (MI5), with ray guns that they shine at him or her at night, then I can test this against my knowledge of MI5 behavior and the likelihood that they are indeed doing this, and it then becomes clear that this experience of my patient is a paranoid persecutory hallucination. If, on the other hand, my patient says that he or she walked into a beautiful garden that was full of the most exotically colored flowers, and says furthermore that this was for him or her a peek into the afterlife, on what grounds can I dispute this, without a set of falsifiable criteria that I can apply to the afterlife?

The next problem I found with Augustine's paper is that he has not looked at the causes of near-death experiences. He treats near-death experiences as if they are a unitary phenomenon. The data, however, 
are very much against that. In our retrospective study of more than 300 NDEs (Fenwick and Fenwick, 1995), near-death experiences occurred when people were relaxing in front of the fire, when they were dreaming at night, spontaneously during the day, or when people were intensely frightened. All these states have underpinning them the normal physiological functioning of the brain, although different systems are involved, such as wakefulness, rapid eye movement (REM) sleep, or intense emotional arousal. NDEs also occurred when people were "ill" (usually not clearly defined), or when people were seriously ill or under anesthesia. All these states, by contrast, have an abnormal brain physiology and a disruption of normal functioning, although the precise details of this would have differed between cases. Ten percent of our cases had their NDEs during the clinical death of cardiac arrest, when the heart had stopped, respiration had ceased, brainstem reflexes were absent, and brain function could not possibly support consciousness. A look at this list does not suggest that there could possibly be a common neurophysiological mechanism. It does suggest that there may be several mechanisms that lead to a common experience, but until this is sorted out it, is impossible to understand the biological/psychological nature of the near-death experience.

This one fact in itself could point towards a common reality that is accessed by different routes, but here again we are back with epistemology and ontology. Testing the "afterlife peek" hypothesis would suggest that peeks into that reality are also almost independent of brain pathology or physiology, so what then is the structure of that reality and how will we know it when we see it? Perhaps we are back again to asking those who have the experience what it means to them. Clearly the way forward is a proper prospective trial where we can have some control over the psychological and physical variables.

\section{Out-of-Body Experiences}

In this section of his paper, Augustine treats out-of-body experiences (OBEs) in different physiological states as if they all had the same physiological mechanism, just as he did with NDEs. What is clear from the literature is that spontaneous out-of-body experiences are very common, that there is no disruption of normal neurophysiological functioning, and that they are probably dissociative states in which the experiencer will gain no veridical perception away from the body. Indeed, as Augustine has pointed out, except in rare cases, tests of 
obtaining "objective" information from this dissociated state usually fail (Murray and Fox, 2005).

The subjective description of the "reality" into which the subject "goes" depends on his or her belief system. These OBEs are not to be confused with experiences of sleep paralysis, which have a different physiological state; the ascending actions of REM sleep; or with those partial OBEs induced by stimulation of the temporal lobe. There are also reports of out-of-body states being associated with confusional states due to operation or illness, or post-ictal confusional states, which again will have separate physiological mechanisms. Doubtless, some of these mechanisms may be present during near-death experiences, but without a clear definition of the cause of the NDE and the relationship of the OBE to it, it is not possible to proceed much further. The most interesting $\mathrm{OBE}$, in my view, is that of the cardiac arrest NDE, when the heart has stopped and the brain is nonfunctional, but yet the experiencer reports an OBE. Here we have a known physiology: severe brain anoxia with electrical silence and an unconscious patient. It is then important to test and to verify objectively whether the experiencer really did have the NDE when unconscious and was able to gain veridical information (Parnia and Fenwick, 2002).

The OBE that is said to occur during cardiac arrest is one situation where near-death experiences can contribute to our understanding of brain-mind mechanism. During cardiac arrest, all the signs of clinical death are present. Yet about 10 percent of patients claim that while they were unconscious they had a near-death experience, and a small proportion of these claim that they had an OBE in which they were able to obtain veridical information, usually about the resuscitation procedure (Greyson, 2003; Schwaninger, Eisenberg, Schechtman, and Weiss, 2002; van Lommel, van Wees, Meyers, and Elfferich, 2001). If their claims could be verified, they would indicate that brain and mind are somehow separate, a hypothesis that could point towards a continuation of consciousness after death, although of course it would not prove it. It would certainly raise challenging questions for our current neuroscience (Parnia, 2007). This is the one experiment that would help us to differentiate the hypothesis of a "peek" into the afterlife by a mind independent of a brain from a dissociative state, which would be impossible in a nonfunctioning brain. Up-to-date, full reviews of theories of consciousness involving mind-brain independence and the evidence for those theories have recently been published 
(Kelly, Kelly, Crabtree, Gauld, Grosso, and Greyson, 2006; Velmans and Schneider, 2007).

In summary, the value of Augustine's paper is that it raises questions about epistemology and ontology in neuroscience. What his paper makes abundantly clear is that many of the putative mechanisms of NDEs have been suggested by authors who have no proper grasp of the width of the field or the phenomena of NDEs, or of the very wide range of mental and physiological states that underpin these experiences. Most could not possibly apply to NDEs as a whole, and many are simplistic, for example, that they are due to sleep paralysis, or that they are due to temporal lobe epilepsy, a confusional state that never has a clear narrative experience. To answer the questions that Augustine raises, a proper prospective clinical trial is required, so that we can gain a proper understanding of brain physiology and psychology at the time of the event, with all the difficulties that this entails.

\section{References}

Beauregard, M. (2007). Mind does really matter: Evidence from neuroimaging studies of emotional self-regulation, psychotherapy, and placebo effect. Progress in Neurobiol. ogy, 81, 218-236.

Fenwick, P., and Fenwick, E. (1995). The truth in the light: An investigation of over 300 near-death experiences. London, England: Hodder Headline.

Fontana, D. (2005). Is there an afterlife? A comprehensive overview of the evidence. Ropley, England: O Books.

Greyson, B. (2003). Incidence and correlates of near-death experiences in a cardiac care unit. General Hospital Psychiatry, 25, 269-276.

Halligan, P. W. (2002). Phantom limbs: The body in the mind. Cognitive Neuropsychiatry, 7, 251-268.

Kelly, E. F., Kelly, E. W., Crabtree, A., Gauld, A., Grosso, M., and Greyson, B. (2006). Irreducible mind: Toward a psychology for the 21st century. Lanham, MD: Rowman and Littlefield.

Murray, C., and Fox, J. (2005). The out-of-body experience and body image: Differences between experients and nonexperients. Journal of Nervous and Mental Disease, 193, 70-72.

Parnia, S. (2007). Do reports of consciousness during cardiac arrest hold the keys to discovering the nature of consciousness? Medical Hypotheses, 69, 933-937.

Parnia, S., and Fenwick, P. (2002). Near death experiences in cardiac arrest: Visions of a dying brain or visions of a new science of consciousness. Resuscitation, 52, 5-11.

Schwartz, J. M., Stapp, H. P., and Beauregard, M. (2005). Quantum physics in neuroscience and psychology: A neurophysical model of mind-brain interaction. Philosophical Transactions of the Royal Society of London, Series B: Biological Sciences, 360, 1309-1327.

Schwaninger, J., Eisenberg, P. R., Schechtman, K. B., and Weiss, A. N. (2002). A prospective analysis of near-death experiences in cardiac arrest patients. Journal of Near-Death Studies, 20, 215-232. 
van Lommel, P., van Wees, R., Meyers, V., and Elfferich, I. (2001). Near-death experience in survivors of cardiac arrest: A prospective study in the Netherlands. Lancet, 358, 2039-2045.

Velmans, M. (2002a). How could conscious experiences affect brains? Journal of Consciousness Studies, 9(11), 3-29.

Velmans, M. (2002b). Making sense of causal interactions between consciousness and brain. Journal of Consciousness Studies, 9(11), 69-96.

Velmans, M., and Schneider, S. (eds.). (2007). The Blackwell companion to consciousness. Oxford, England: Blackwell Publishing. 